\title{
Experimental Study on the Thermal Argon Plasma Generation and Jet Length Change Characteristics at Atmospheric Pressure
}

\author{
Wenxia Pan · Xian Meng · Xi Chen · Chengkang Wu
}

Received: 16 June 2005/Accepted: 8 December 2005 /

Published online: 24 May 2006

(C) Springer Science+Business Media, Inc. 2006

\begin{abstract}
The generation, jet length and flow-regime change characteristics of argon plasma issuing into ambient air have been experimentally examined. Different torch structures have been used in the tests. Laminar plasma jets can be generated within a rather wide range of working-gas flow rates, and an unsteady transitional flow state exists between the laminar and turbulent flow regimes. The high-temperature region length of the laminar plasma jet can be over an order longer than that of the turbulent plasma jet and increases with increasing argon flow rate or arc current, while the jet length of the turbulent plasma is less influenced by the generating parameters. The flow field of the plasma jet has very high radial gradients of plasma parameters, and a Reynolds number alone calculated in the ordinary manner may not adequately serve as a criterion for transition. The laminar plasma jet can have a higher velocity than that of an unsteady or turbulent jet. The long laminar plasma jet has good stiffness to withstand the impact of laterally injected cold gas and particulate matter. It could be used as a rather ideal object for fundamental studies and be applied to novel materials processing due to its attractive stable and adjustable properties.
\end{abstract}

Keywords Thermal plasma - Generating parameter - Laminar jet - Turbulent flow · Jet-length change

\section{Introduction}

The research and application of DC non-transferred arc plasma jets have had a rather long history. Various plasma jets with different flow field characteristics and generated by use of different plasma torches have been employed to meet various demands raised in science and engineering. The main application area of thermal plasma jets was the space technology in the 60 s and 70s of the last century, generally used for aerodynamic heating simulation [1]. These plasma jets were usually of high power, high thermal efficiency, high pressure, large

W. X. Pan $(\varangle) \cdot$ X. Meng · C. K. Wu

Institute of Mechanics, Chinese Academy of Sciences, Beijing 100080, China

e-mail:wxpan@imech.ac.cn

Xi Chen

Department of Engineering Mechanics, Tsinghua University, Beijing 100084, China 
flow rate and relatively large sizes; and thus, almost inevitably, the jet flow was turbulent. From the 80 s of the last century, the application of thermal plasma jets (especially turbulent plasma jets) in materials processing has been rapidly increasing [2, 3]. Using turbulent jets in materials processing, however, has some inherent disadvantages, such as worsen working surroundings and poor process controllability and reproducibility, due to the intensive fluctuation of the turbulent plasma jet, high-intensity noise emission, and heavy entrainment of the ambient air into the jet [2-4] and thus serious oxidation of metallic materials processed in air surroundings. Those disadvantages make the usage of turbulent plasma jets be limited to materials processing with lower precision requirement. Nevertheless, so far the turbulent DC arc plasma jets are still widely used for materials processing, and many new type DC arc plasma torches, such as the multi-cathode plasma torch, the multi-jet plasma generator etc., have been developed to generate turbulent plasma jets for various special applications.

Recently long laminar plasma jets have been successfully generated using DC nontransferred arc plasma torches at atmospheric pressure with a relatively simple modification of the plasma torch design, i.e. adding a piece of inter-electrode insert between the cathode and the anode [5]. Studies indicate that the long laminar plasma jets possess stable flow state, rather high flow velocity (hard or directional flow characteristic), negligible noise emission, appreciably reduced entrainment of ambient air and adjustable jet length, and thus are favorable for achieving good process reproducibility and controllability [5-8]. Preliminary attempts $[7,8]$ to use the long laminar plasma jets in the preparation of thermal barrier coatings, in the re-melting hardening of cast iron surfaces and in the stainless-steel surface cladding have shown encouraging results, including the fine microstructure, low surface roughness and small porosity of the prepared coatings, the good re-melting process controllability and surface morphologies, as well as the preferable metallurgically bonded clad layer.

There have been many studies of turbulent plasma jets, e.g. see Refs. [2-4] and the references therein. On the other hand, although a number of papers [5-17] have been published to study the generation and characterization of the laminar plasma jets and to explore its applications in materials processing, so far not enough attention has been paid on the study of laminar plasma in the thermal plasma science and technology community. Reasons in two respects might be responsible for this situation hindering the development and application of laminar plasma jets. The first one could be due to the difficulty encountered in the generation of usable and stable long laminar plasma jets, and the second one could be due to the general inference, although without the support of convincing evidence, that the laminar plasma jet always has too low flow momentum, easily disturbed flow characteristics and too low energy efficiency of the plasma torch.

Up to now there is no systematic study to compare the laminar and turbulent thermal plasma jet characteristics. A series of experiments are thus conducted to study the thermal plasma jet generation and the jet length change characteristics in order to provide basic support for the novel process design using the laminar plasma jets. Different plasma torch structures are tested to examine their effects on the generated plasma jets. The effects of laterally injected material powder and its carrier gas on the laminar plasma jet are also investigated. It is our hope that this paper may arouse more interest in the study of long laminar plasma jet characteristics and in their practical applications to materials processing.

\section{Experiments}

The DC non-transferred arc plasma torches with an internal structure similar to that employed in our previous work [5] are used in the present experimental study. The anode-nozzle is of 
$4 \mathrm{~mm}$ inner-diameter. Pure argon is used as the working gas with mass flow rates in the range of $1.6 \times 10^{-4}-5.5 \times 10^{-4} \mathrm{~kg} / \mathrm{s}$, and the plasma jet is issuing into the atmospheric-pressure air surroundings. The argon is admitted into the plasma torch axially and tangentially around the upstream end of the cathode and also tangentially into the torch from an annular slot near the downstream section of the inter-electrode insert. The arc currents are in the range of 160-220 A. In order to examine the effects of the torch structure on the generated plasma jets, two inter-electrode inserts of larger $(4 \mathrm{~mm})$ and smaller $(3 \mathrm{~mm})$ central-hole diameters, will be called $D_{\mathrm{L}}$ and $D_{\mathrm{S}}$ respectively, are used to construct the plasma torch while other structure of the plasma torch is kept unchanged. Photographs of the appearance of the horizontal issuing jets are taken from lateral/horizontal direction to determine the visual high-temperature region length of the plasma jet (will be called the jet length) and to observe whether or not natural convection affects the jet appearance. In a few experiments, alumina powder with particle sizes less than $30 \mu \mathrm{m}$ is laterally injected into the laminar plasma jet with argon as its carrier gas in order to examine whether the laminar plasma jet can withstand the impact by laterally injected particulate matter and its carrier gas and to observe the effect of the injected powder and carrier-gas on the laminar plasma jet length.

\section{Results and discussion}

\subsection{Dependence of jet flow regimes and jet lengths on generating parameters}

Figure 1 presents the experimental results concerning the jet flow states and the visual jet lengths obtained from the photographs by use of the plasma torch with 3-mm-diameter interelectrode insert $\left(D_{\mathrm{S}}\right)$. In this figure the measured jet lengths are indicated by numerals (in $\mathrm{mm}$ ) for different argon mass flow rates and different arc currents. It is found that there exist three distinct flow regimes of the plasma jets, i.e. the laminar, turbulent and transitional regimes, with quite different jet characteristics, depending on the process parameters (arc current and argon flow rate). For a fixed arc current, noisy, fluctuating and short turbulent plasma jets are produced at higher gas flow rates, and the jet lengths of the turbulent plasma jets are less dependent on the gas flow rate or the arc current. On the other hand, quiet, stable and long laminar plasma jets are generated at lower gas flow rates, and the jet length of the laminar plasma jet increases with increasing gas flow rate for a fixed arc current and increases with increasing arc current for a fixed gas flow rate. Between the turbulent and laminar flow regimes, i.e. within the transitional flow regime, the plasma jets are unsteady and accompanied with rather large magnitude fluctuation in the jet length and in the jet noise intensity (the noise intensity increases as the jet length shortens and decreases as the jet length elongates). Hence, the jet lengths in the transitional region indicated in Fig. 1 are their time-averaged values, and the average jet length decreases with increasing gas flow rate for a fixed arc current but increases with increasing arc current for a fixed gas flow rate. The experimental data presented in Fig. 1 demonstrate that the long laminar plasma jets can be generated in a rather wide range of working parameters of the plasma torch, and increasing the arc current allows the laminar plasma jet able to be generated at higher gas flow rates.

Two straight lines have been added in Fig. 1 to separate the different flow regimes of the plasma jets. The solid line on the left side represents the critical condition for the transition from the stable long laminar plasma jet regime into the unsteady transitional flow regime. This solid line shows that the critical flow rate for the laminar plasma jet able to be generated increases with increasing arc current, and can be expressed by the empirical relation 
Fig. 1 Variations of the jet flow state and jet length with the argon mass flow rate and arc current. Torch $D_{\mathrm{S}}$ is used. The numerals in the figure indicate the measured visual jet lengths in $\mathrm{mm}$.

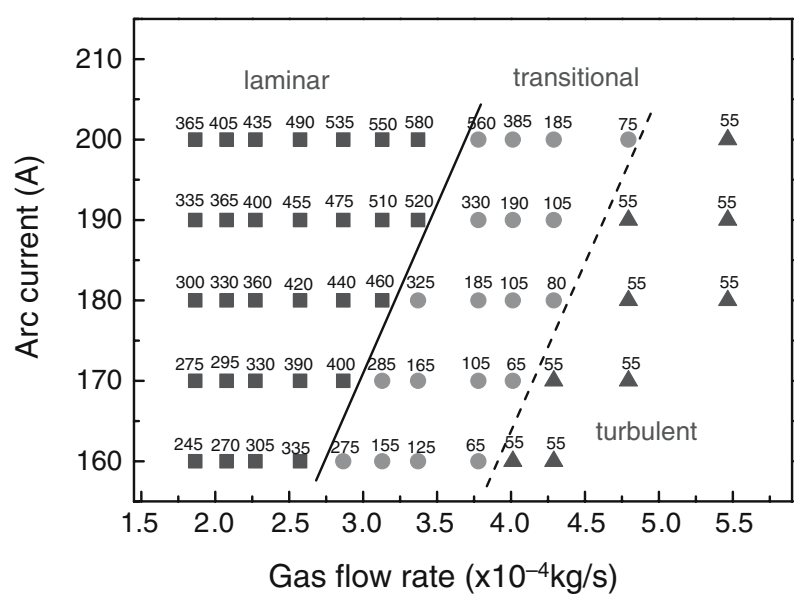

$G_{\mathrm{L}}=0.024 I-1.10$

where $I$ is the arc current (in A) and $G_{\mathrm{L}}$ is the $\operatorname{argon}$ mass flow rate (in $10^{-4} \mathrm{~kg} / \mathrm{s}$ ) at which the laminar plasma jet assumes its maximum length for the given arc current $I$. The dashed line on the right side in Fig. 1 represents the critical condition for the transition from the unsteady transitional flow regime into the short turbulent plasma jet regime. This dashed line is almost parallel to the solid line, and can be expressed by the empirical relation

$$
G_{\mathrm{T}}=0.024 I+0.07
$$

where $I$ is still the arc current in A, whereas $G_{\mathrm{T}}$ is the minimum argon mass flow rate (in $10^{-4} \mathrm{~kg} / \mathrm{s}$ ) at which the noisy short turbulent jet will be produced for the given arc current $I$. Both the critical flow rates $G_{\mathrm{L}}$ and $G_{\mathrm{T}}$ are the linearly increasing functions of the arc current $I$. For a given arc current, there are only one $G_{\mathrm{L}}$ value, corresponding to the maximum possible gas flow rate for the laminar plasma jet able to be generated, and one $G_{\mathrm{T}}$ value, corresponding to the minimum possible gas flow rate for the turbulent plasma jet to be produced.

\subsection{Arc voltage-current characteristics}

For the same plasma torch as used for Fig. 1, Fig. 2 shows the variation of the arc voltage (measured between the torch anode and cathode) with arc current for several different argon mass flow rates. The data denoted by solid marks in Fig. 2 correspond to the long laminar jet regime, half-hollow marks to the transitional regime, while the hollow marks correspond to the short turbulent jet regime. It is seen that the arc voltage increases linearly with increasing arc current for a fixed argon mass flow rate. Figure 3 shows the variation of the arc voltage with the argon mass flow rate for three different arc currents. It is seen that for a fixed arc current, the arc voltage increases approximately linearly with increasing argon flow rate for this plasma torch, although different flow regimes are all involved. Two inclined straight lines are also added in Fig. 3 to express the critical conditions for the flow-state transition of the plasma jet, similar to the two critical lines shown in Fig. 1.

The increasing voltage-current characteristics shown in Fig. 2 are typical for a wallstabilized or constricted arc. The fact that the arc voltage increases approximately linearly with increasing argon flow rate, regardless of the different jet flow regimes, seems to indicate 
Fig. 2 Measured voltage-current characteristics for the plasma generation using the torch $D_{\mathrm{S}}$. Solid marks correspond to the laminar jet regime, half-hollow and hollow marks to the turbulent jet regime marks to the transitional regime

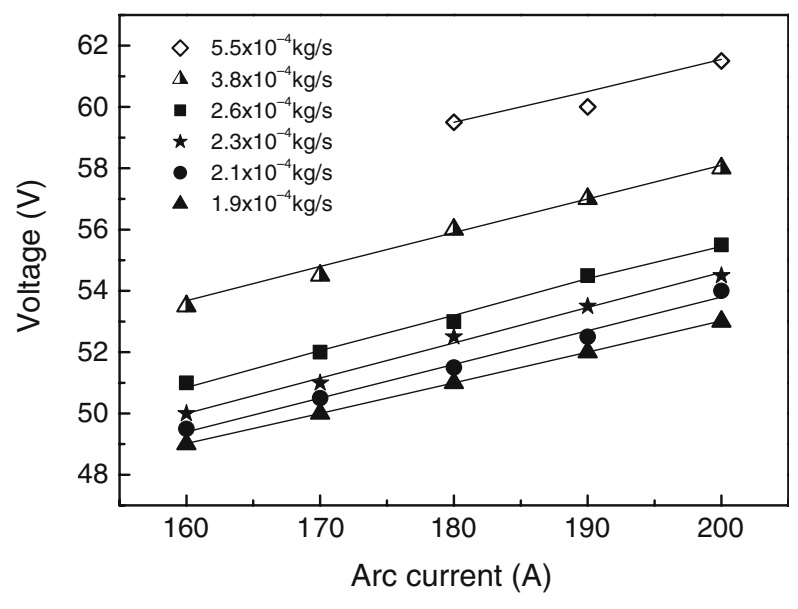

Fig. 3 Variations of the arc voltage with the argon flow rate using the torch $D_{\mathrm{S}}$

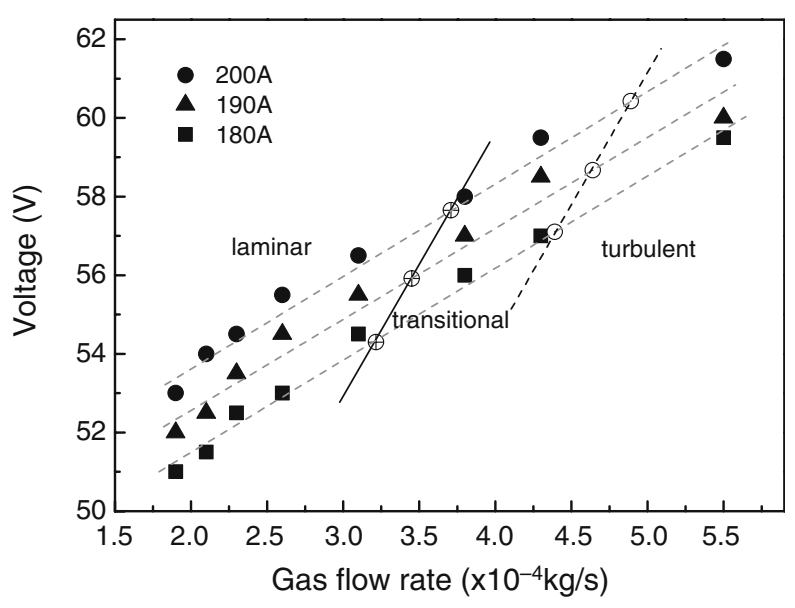

that the working character of the arc column within the plasma torch is almost the same for jet generation in all the jet flow regimes, implying that the transition of the jet flow state occurs only in the downstream region beyond the arc-root attachment point on the anode surface.

\subsection{Effects of inter-electrode insert diameter on the jet characteristics}

Employing the plasma torch with the inter-electrode insert of 4-mm hole-diameter (i.e. $D_{\mathrm{L}}$, instead of $D_{\mathrm{S}}$ used for Figs. 1-3) for generating the plasma jets, experimental results are shown in Fig. 4 for the variations of the flow state and jet length with argon mass flow rate and arc current. The numerals in Fig. 4 also denote the measured visual jet lengths in $\mathrm{mm}$. It is seen that the experimental results presented in Fig. 4 are generally similar to those shown in Fig. 1 obtained for the case using the plasma torch with the inter-electrode insert of 3-mm hole-diameter $\left(D_{\mathrm{S}}\right)$. Nevertheless, the details of experimental data shown in Fig. 4 are somewhat different from those in Fig. 1. For example, the two straight lines representing the critical conditions for the transition from the long laminar plasma jet regime to the unsteady transitional flow regime and from the unsteady transitional flow regime to the short turbulent 
Fig. 4 Variations of the flow regime and jet length with the argon flow rate and arc current. Torch $D_{\mathrm{L}}$ is used. The numerals in the figure indicate the measured visual jet lengths in $\mathrm{mm}$

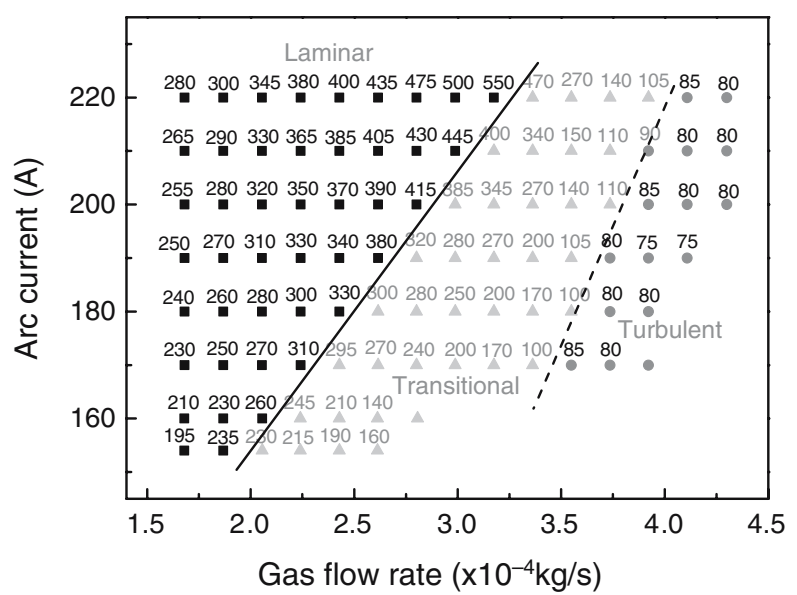

plasma jet regime are no longer parallel in Fig. 4. The left critical line now can be expressed by the following relation:

$$
G_{\mathrm{L}}=0.019 I-0.92
$$

For the present case, transition from the long laminar plasma jet regime to the unsteady transitional flow regime occurs at argon mass flow rate of $2.12 \times 10^{-4} \mathrm{~kg} / \mathrm{s}$ for arc current of $160 \mathrm{~A}$, while the transition flow rate increases to $3.26 \times 10^{-4} \mathrm{~kg} / \mathrm{s}$ for arc current of $220 \mathrm{~A}$. It is also seen that the transitional flow region becomes narrower with the increase of the arc current for the present case.

Figure 5 compares the jet length variations with argon mass flow rate for the two plasma torches with different inter-electrode-insert central-hole diameters (i.e. 3 and $4 \mathrm{~mm}$ ) for two different arc currents (170 and 190 A), while Fig. 6 compares corresponding jet length variations with arc current for three different gas flow rates (i.e. 1.9, 2.1 and $2.3 \times 10^{-4} \mathrm{~kg} / \mathrm{s}$ ). It is seen clearly from Fig. 5 and Fig. 6 that when the inter-electrode insert with a smaller central-hole diameter is used for the plasma jet generation, the laminar jet regime can be obtained at higher gas flow rates and longer laminar jets can be generated. Figure 7 compares the jet length variations with the torch input power for two different gas flow-rates (i.e. 2.1 and $2.3 \times 10^{-4} \mathrm{~kg} / \mathrm{s}$ ) obtained by use of the two plasma torches with different interelectrode-insert hole diameters. It is seen that when the inter-electrode insert with a smaller hole diameter $\left(D_{\mathrm{S}}\right)$ is used for the plasma jet generation, longer laminar plasma jets than the case $D_{\mathrm{L}}$ can be produced for the same input power and gas flow rate. It seems that the jet issuing from the $D_{\mathrm{S}}$ torch has higher net power and higher central temperature and velocity than those from the $D_{\mathrm{L}}$ torch for a given arc current, perhaps due to more intense constriction of the arc column in the inter-electrode insert $D_{\mathrm{S}}$.

It is interesting to note that in the above experiments, only the diameter of the interelectrode insert is changed ( $3 \mathrm{vs} .4 \mathrm{~mm}$ ) and all the other sizes are kept the same for the two torches including their anode-nozzle inner-diameter $(4 \mathrm{~mm})$. Hence, when the arc column is restricted to the flow channel within the inter-electrode insert of smaller hole-diameter, the argon flow will be accelerated to a higher velocity in comparison with the case using the larger-hole-diameter insert for the same working-gas flow rate and arc current. The experimental results presented in Fig. 5 show clearly that employing the smaller-hole-diameter insert allows the jet to keep its laminar flow state at higher gas flow rate than that for the case 
Fig. 5 Comparison of the flow-regime and jet length change characteristics for the plasma jets generated using different torches $D_{\mathrm{L}}$ and $D_{\mathrm{S}}$

Fig. 6 Comparison of the jet length variations with the arc current for the laminar plasma jets generated using different torches $D_{\mathrm{L}}$ (hollow symbols) and $D_{\mathrm{S}}$ (solid symbols)
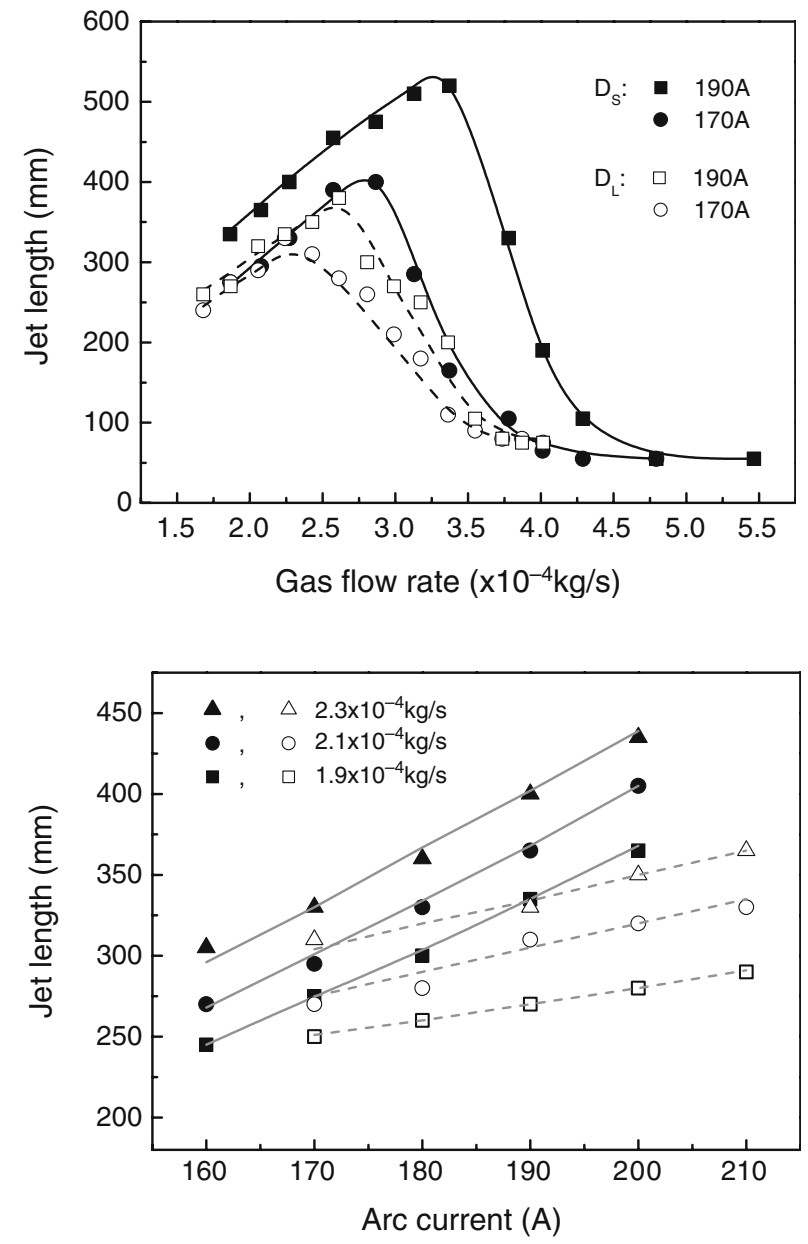

Fig. 7 Jet length variations with the input power for the laminar plasma jets generated using different torches $D_{\mathrm{L}}$ (hollow symbols) and $D_{\mathrm{S}}$ (solid symbols)

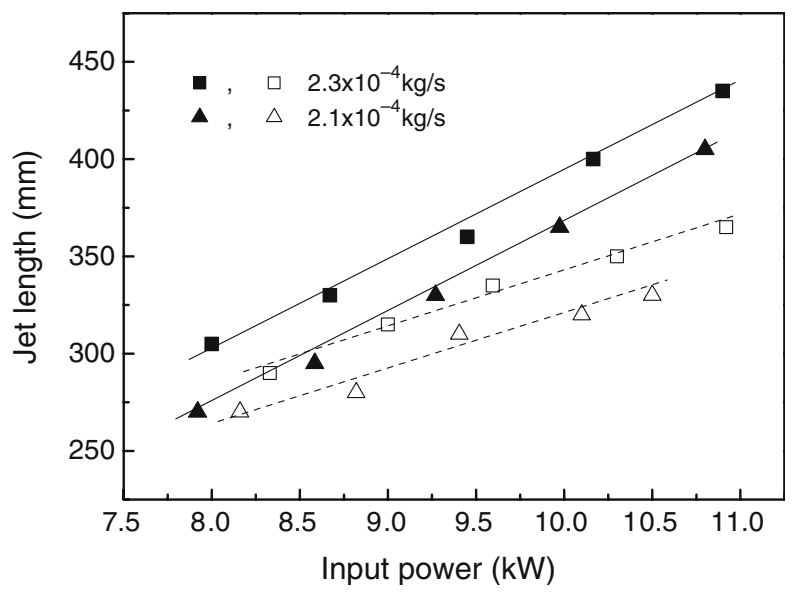


employing the lager-hole-diameter insert for a given arc current. Thus, the results in Figs. 1, 4 and 5 tell us an important fact that the conventional viewpoint, which considers the laminar plasma jet should certainly have a lower velocity than that for an unsteady or turbulent jet, is not always correct for the laminar plasma generation. Elevation of the plasma temperature and velocity in the insert can increase, to a certain extent, the ability for the laminar plasma jet to resist the external disturbance in the jet generation.

3.4. Ability of the laminar jet to withstand the impact of the lateral injected powder and carrier-gas and the AC disturbance

Figure 8 presents four photographs of the laminar plasma jets for the cases (a) without injection of carrier gas (argon) and $\mathrm{Al}_{2} \mathrm{O}_{3}$ powder; (b)-(d) with lateral injection of the $\mathrm{Al}_{2} \mathrm{O}_{3}$ powder and its carrier gas, and the carrier-gas flow rates are (b) $0.82 \times 10^{-5} \mathrm{~kg} / \mathrm{s}$; (c) $1.90 \times$ $10^{-5} \mathrm{~kg} / \mathrm{s}$ and (d) $3.26 \times 10^{-5} \mathrm{~kg} / \mathrm{s}$, respectively. The mass flow rate of the injected $\mathrm{Al}_{2} \mathrm{O}_{3}$ powder increases with the carrier-gas flow rate, although the accurate values of the injected $\mathrm{Al}_{2} \mathrm{O}_{3}$ powder flow rate have not been measured. The white line or smog from the feeding nozzle to or around the plasma jet in the pictures just shows the situation that the $\mathrm{Al}_{2} \mathrm{O}_{3}$ powder is fed. It can be seen from Fig. 8(b), (c) and (d) that the long laminar plasma jet has enough stiffness to withstand the impact of the laterally injected powder and its carrier gas, in agreement with the modeling result given in Ref. [12]. The plasma jet still retains its laminar flow state even for the case with lateral injection of the powder and its carrier gas according to the experimental observation. The effect of natural convection on the appearance of the long laminar plasma jets horizontally issuing into ambient air is negligibly small, in agreement with the modeling result given in Ref. [17]. It is expected that the jet energy consumption in heating the $\mathrm{Al}_{2} \mathrm{O}_{3}$ powder and its carrier gas will lead to the decrease of plasma temperature or the shortening of the laminar plasma jet length. The more the carrier gas and powder feeding into the plasma jet are, the shorter the laminar plasma jet length will be. The experimental observation shown in Fig. 8(b) (c) and (d) demonstrates this expectation. The situation of Fig. 8(d) is actually an extreme condition under which large amount of powder abruptly rushes into the laminar plasma jet. Those powder particles are previously accumulated in the feeding-tube passage at lower carrier-gas flow rate and then are unexpectedly blown out at the higher carrier-gas flow rate. It is interesting to note that the stiffness of the laminar plasma jet is still good even for this extreme case.

The experimental results presented in Ref. [16] show that due to the existence of AC components in the DC power supply employed in the experiment, there exist $300 \mathrm{~Hz}$ regular fluctuations in the arc voltage and in the jet impact pressure measured near the torch exit center even for the laminar plasma jets. That is, there always exists external disturbance in the plasma jet generation. However, it is found that the laminar plasma jets can still be produced and can maintain their laminar flow state.

\subsection{Additional discussion}

In fluid mechanics, Reynolds number $R e=\rho u D / \mu$ is usually employed as the criterion to judge the flow state (laminar or turbulent) for a round gas jet. Thus one might suspect that there could be a more or less equal value of the Reynolds number along the solid critical lines in Figs. 1 and 4 at which the transition from the laminar flow state to the unsteady transitional flow state happens. For the present case with great radial variations of plasma temperature and velocity, the mass flow rate $G$ in the plasma torch can be expressed as $G=(\pi / 4) D^{2} \rho u$ (where $D, \rho$ and $u$ are the anode-nozzle inner-diameter and average gas density and velocity, 


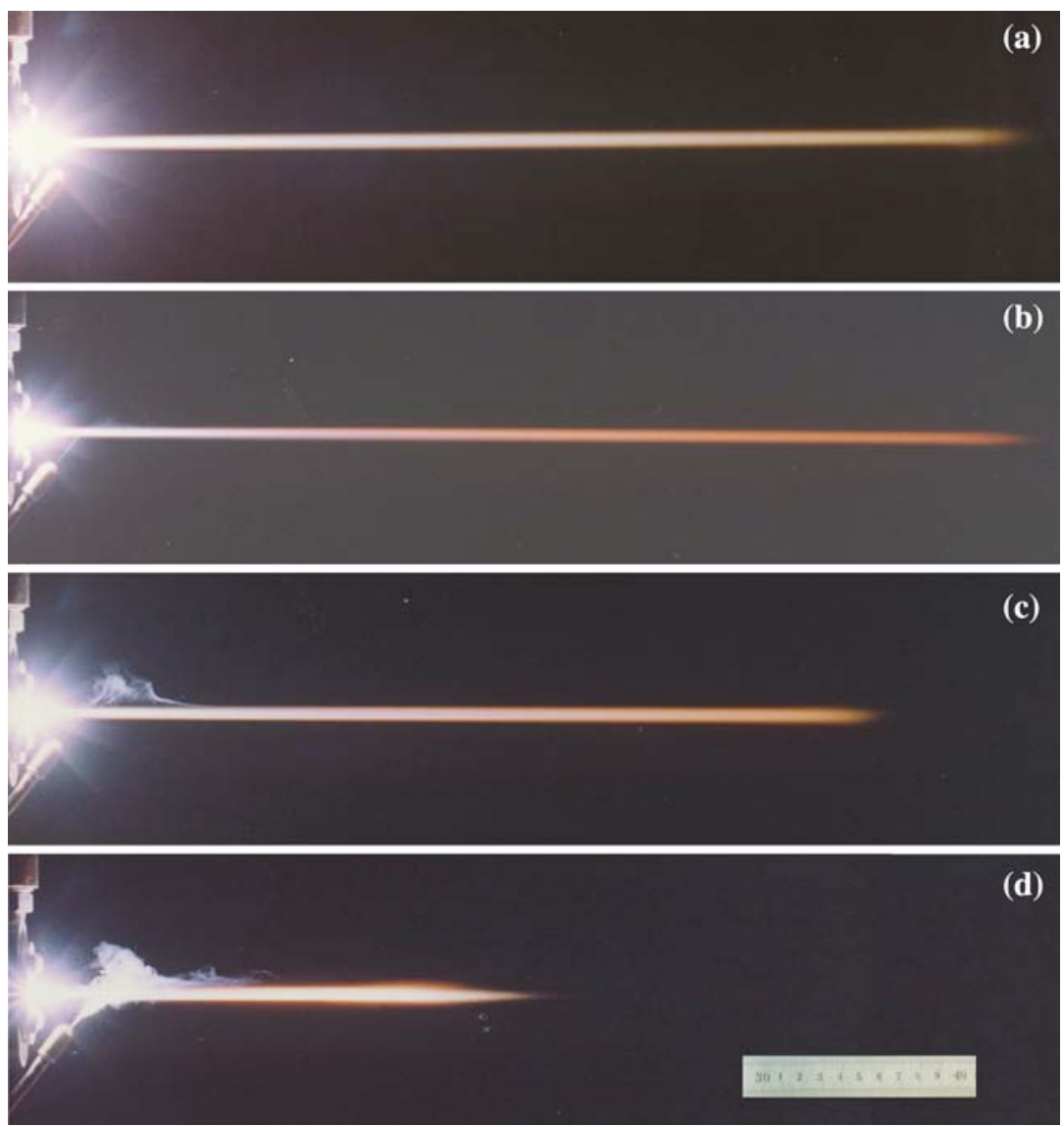

Fig. 8 Photographs of laminar plasma jets for the cases (a) without powder and carrier-gas injection, (b) $-(\mathbf{d})$ with lateral injection of $\mathrm{Al}_{2} \mathrm{O}_{3}$ powder and its carrier gas, and the carrier-gas flow rate is (b) $0.82 \times 10^{-5} \mathrm{~kg} / \mathrm{s}$, (c) $1.90 \times 10^{-5} \mathrm{~kg} / \mathrm{s}$, and (d) $3.26 \times 10^{-5} \mathrm{~kg} / \mathrm{s}$

respectively) and thus $R e=4 G /(\pi D \mu)$ can be used to calculate the Reynolds number with the merit that $G$ and $D$ are known unambiguously and only the viscosity $\mu$ is to be determined at a suitably chosen characteristic temperature. Several different choices of the characteristic temperature were proposed in previous studies. For example, Ref. [18] calculated $R$ e by using the gas viscosity at the temperature corresponding to the mass-averaged enthalpy at the torch exit (i.e. $R e_{\mathrm{av}}$ ), and correlated the $R e_{\mathrm{av}}$ with the dimensionless axial-location at which an originally 'laminar' jet transited into a turbulent state. On the other hand, Ref. [19] used an effective Reynolds number $\operatorname{Re}_{\mathrm{eff}}\left(=R e_{\mathrm{av}} \times \Gamma_{\mathrm{av}}\right)$ to judge the flow state (laminar or turbulent) for a fully developed arc in a water-cooled cylindrical tube, where the factor $\Gamma_{\mathrm{av}}$ was introduced to account for the radial variation of plasma properties at the cross section of the fully developed arc. In the present experiment the arc root is found to be attached at a location near the upstream end of the anode-nozzle, and this situation is obviously different from those studied in $[18,19]$. Employing the voltage -ampere characteristics shown in Figs. 2 and 3 and the measured torch efficiency [11] and taking the temperature corresponding to the mass-averaged specific-enthalpy at the exit of anode-nozzle as the characteristic 
temperature to evaluate the gas viscosity, it is found that along the solid line in Fig. 1, the values of $R e_{\text {av }}$ are 331, 389 and 448, respectively, corresponding to arc current 160, 180 and 200 A. Since the flow instability should begin in the shear layer near the torch wall or near the jet boundary, we also try to use a certain temperature within the thermal boundary layer, i.e. $T_{\text {ref }}=T_{\mathrm{w}}+\alpha\left(T_{\mathrm{m}}-T_{\mathrm{w}}\right.$ ) (where $T_{\mathrm{w}}$ and $T_{\mathrm{m}}$ are the torch wall or the ambient air temperature and the jet peak temperature [13], and $\alpha$ is an adjustable parameter, respectively), as the characteristic temperature to calculated $R e$. The obtained values of $R e$ along the solid line in Fig. 1 are 849, 979 and 1104, respectively, for the arc currents of 160, 180 and 200 A when $\alpha=0.1$ is chosen, whereas the calculated $R e$ values become 613,703 and 789, respectively, for $\alpha=0.2$. Although these $R e$ values are reasonable, the calculated critical Reynolds numbers along the solid line in Fig. 1 are not constant no matter which characteristic temperature is applied. This fact shows the complexity of thermal plasma jets and thus further research effort is required in future to achieve the generalization of critical Reynolds number.

It is anticipated that the long laminar plasma jet can become a rather ideal object of fundamental studies of thermal plasmas due to non-existence of the complexity caused by the turbulence and its stable and adjustable flow characteristics. For example, comparative measurements of the temperatures profiles of laminar and turbulent jets can be helpful to clarify different non-equilibrium characteristics in the edge regions of the two kinds of plasma jets. The long laminar plasma jets may also find their position in materials processing due to their features of low noise emission and better process controllability and repeatability, although smaller jet diameter $(\sim 4 \mathrm{~mm})$ and great radial gradients of plasma parameters require paying more attention to control the powder injection and the particle confinement if powder processing is involved.

This paper mainly presents the experimental results concerning the dependence of the plasma jet flow state and the jet length on the torch parameters (working-gas flow rate and arc current). In order to obtain better understanding of the laminar plasma jet characteristics, still further studies are required. For example, spatially resolved measurements of plasma temperature, velocity and species concentration distributions within the laminar plasma jet should be taken as one of the subjects of subsequent studies.

\section{Conclusions}

Both laminar and turbulent plasma jets can be generated at atmospheric pressure by use of the same DC non-transferred arc plasma torch. The experimental results show that stable, silent and long laminar plasma jets are generated at lower argon flow rates, while fluctuating, noisy and short turbulent plasma jets are produced at higher gas flow rates. Between the laminar and turbulent flow regimes, there exists an unsteady transitional regime. The hightemperature region length of laminar plasma jet is much longer than that of the turbulent plasma jet, and the laminar jet length increases with argon flow rate or arc current. On the other hand, the turbulent plasma jet length depends less on the working-gas flow rate or the arc current. Reynolds number alone calculated in the ordinary way may not be an adequate criterion for flow transition for such complicated flow fields. The plasma torch structure (insert hole-diameter) somewhat affects the generated jet characteristics. The long laminar plasma jet has good stiffness to withstand the impact of laterally injected particulate matter and its carrier gas, and the effect of natural convection on the laminar plasma jet appearance can be ignored. The laminar plasma jet can be generated within a rather wide range of gas flow rates and arc currents. It could be useful in fundamental studies due to non-existence of 
the complexity caused by turbulence and its stable and adjustable flow characteristics, and could find its position in novel materials processing due to its marked advantages.

Acknowledgment This work was supported by the National Natural Science Foundation of China (Nos. 50336010, 50276065).

\section{References}

1. Pfender E (1978) In: Hirsh MN, Oskam HJ (eds.) Electrical discharges, Academic Press, New York, pp 291-398

2. Pfender E (1999) Plasma Chem Plasma Process 19:1

3. Fauchais P (2004) J Phys D: Appl Phys 37:R86

4. Pfender E, Spores R, Chen WLT (1995) Int J Mater Product Technol 10:548

5. Pan WX, Zhang WH, Zhang WH, Wu CK (2001) Plasma Chem Plasma Process 21:23

6. Pan WX, Li G, Meng X, Ma W, Wu CK (2005) Pure Appl Chem 77:373

7. Pan WX, Meng X, Li G, Fei QX, Wu CK (2005) Surf Coatings Tech. 97:345

8. Pan WX, Ma W, Wu CK (2001) In: Zhou Y-C, Gu Y-X, Li Z (eds.) Proceedings of mechanics and material engineering for science and experiments, Science Press, Beijing, pp 427-431

9. Solonenko OP (1994) In: Solonenko OP, Zhukov MF (eds.) Thermal plasma and new materials technology Vol. 2. Cambridge Interscience Publishing, Cambridge, pp 7-96

10. Osaki K, Fukumasa O, Kobayashi A (2000) Vacuum 59:47

11. Pan WX, Zhang WH, Ma W, Wu CK (2002) Plasma Chem Plasma Process 22:271

12. Xu D-Y, Chen Xi, Cheng K (2003) J Phys D: Appl Phys 36:1583

13. Meng X, Pan WX, Wu CK (2003) In: Proceedings of the 16th International Symposium on Plasma Chemistry, Taomina, Italy, Paper No. ISPC-243

14. Cheng K, Chen Xi (2004) J Phys D: Appl Phys 37:2385

15. Li G, Pan WX, Meng X, Wu CK (2005) Plasma Sources Sci Technol 14:219

16. Meng X, Pan WX, Li T, Wu CK (2005) In: Proceedings of the 17th International Symposium on Plasma Chemistry, Toronto, Paper No. ISPC-354

17. Xu D-Y, Chen Xi, Pan WX (2005) Int J Heat Mass Transfer 48:3253

18. Fincke JR, Pentecost CG (1991) In: Etemadi K, Mostaghimi J (eds.) Heat transfer in thermal plasma processing, ASME HTD-161, pp 101-106

19. Sinkevich OA, Chikunov SE (2004) Fluid Dynamics 39:718 